\title{
Methylation-regulated ZNF545 inhibits growth of the p53-mutant KYSE150 cell line by inducing p21 and Bax
}

\author{
YU FAN ${ }^{1}$, YU WANG ${ }^{2}$, SHAOZHI FU $^{1}$, DUAN LIU $^{1}$ and SHENG LIN ${ }^{1}$ \\ Departments of ${ }^{1}$ Oncology and ${ }^{2}$ Health Examination, \\ The Affiliated Hospital of Southwest Medical University, Luzhou, Sichuan 646000, P.R. China
}

Received October 18, 2018; Accepted May 2, 2019

DOI: $10.3892 /$ etm.2019.7737

\begin{abstract}
The Krüppel-associated box zinc-finger protein 545 (ZNF545) was recently identified as a tumor suppressor in esophageal squamous cell carcinoma (ESCC). However, the role of ZNF545 in the tumorigenesis of esophageal cancer cells expressing loss-of-function mutant p53 has not been elucidated. In the present study, the role of ZNF545 in esophageal tumors and the p53-mutant ESCC cell line, KYSE150, was investigated. ZNF545 mRNA was significantly downregulated in tumors when compared with adjacent normal tissues. Methylated ZNF545 was detected in $76.6 \%$ of tumor tissues compared with $28.1 \%$ of adjacent normal tissues. Combined pharmacological treatment of KYSE150 cells with a demethylating reagent and deacetylase inhibitor restored the expression of ZNF545. Ectopic expression of ZNF545 activated p53 transcription and upregulated the protein expression levels of pivotal effectors p21 and Bax, which are associated with cell proliferation and apoptosis, respectively, in p53-mutant KYSE150 cells; while suppressing colony formation and inducing apoptosis. ZNF545 was therefore proposed as a potential tumor suppressor responsible for inhibiting the growth of p53-mutant ESCC cell lines. In addition, tumor-specific methylation of ZNF545 may represent an epigenetic diagnostic biomarker and a therapeutic target in patients with esophageal cancer.
\end{abstract}

\section{Introduction}

Esophageal cancer is a common malignancy of the digestive tract, with the sixth-highest cancer associated mortality rate worldwide (1). Esophageal squamous cell carcinoma (ESCC) is particularly prevalent in far-eastern countries including China (2). The 5-year survival rates of patients with

Correspondence to: Dr Yu Fan, Department of Oncology, The Affiliated Hospital of Southwest Medical University, 25 Taiping Street, Jiangyang, Luzhou, Sichuan 646000, P.R. China

E-mail: yufan@swmu.edu.cn

Key words: esophageal squamous cell carcinoma, zinc-finger protein 545, methylation, tumor suppressor gene, mutant p53 early esophageal cancer has improved drastically as a result of multidisciplinary developments in treatment strategies; however, the risk of mortality from advanced esophageal cancer remains high.

Aberrant inactivation of tumor suppressor genes by promoter methylation is frequently involved in tumorigenesis. In particular, there has been accumulating evidence reveling a close association between methylated tumor suppressor-encoding genes and the development and progression of malignant tumors (3). Zinc finger proteins (ZFPs) are the largest family of protein transcription factors in mammals. A Krüppel-associated box zinc-finger protein, ZFP82/ZNF545/KIAA1948 is considered to be a novel tumor suppressive gene that is frequently downregulated and methylated in multiple carcinomas including in breast and gastric cancer (4). Furthermore, the biological functions of ZNF545 are reported to be involved in cell proliferation and apoptosis, and serve an important role in cancer development. It has been demonstrated previously that ZNF545 suppresses tumor progression by inhibiting nuclear factor $-\kappa \mathrm{B}$ activation in ESCC cell lines; however, it is frequently methylated in ESCC $(4,5)$. In addition, another previous study demonstrated that ZNF545 may be involved in regulating p53 signaling in multiple myeloma (6).

Frequent point mutations in the tumor suppressor gene p53 have been identified in both primary ESCCs and in ESCC cell lines. Point mutations in this gene may occur at early stages of ESCC and correlate with tumor progression, indicating that they may serve an important role in ESCC. However, the role of ZNF545 remains unclear in p53-mutant ESCC cell lines.

Based on the role of ZNF545 in ESCC, the effect of ZNF545 overexpression on p53 gene expression and its downstream signaling pathways in the p53-mutant ESCC cell line, KYSE150, was investigated. In addition, the clinical significance of ZNF545 expression in patients with ESCC was examined.

\section{Materials and methods}

Tissue specimens, cell line and plasmid transfection. Fresh ESCC and adjacent normal tissues were obtained from

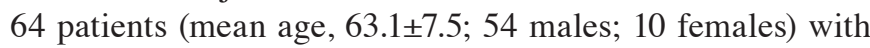
no second primary malignant tumor that had undergone an esophagectomy at the Department of Cardiothoracic Surgery, 
the Affiliated Hospital of Southwest Medical University (Luzhou, China) from March 2015 to February 2016. All patients were newly diagnosed and had not received treatment prior to the study. Written informed consent was provided by all patients and the clinical and pathological data were obtained. The current study was approved by the Institutional Ethics Committees of the Affiliated Hospital of Southwest Medical University, and followed the principles of the Declaration of Helsinki. The esophageal cancer cell line, KYSE150, was anonymously donated by a professor from Chongqing Medical University (Chonqing, China) and was cultured in RPMI 1640 medium (Gibco; Thermo Fisher Scientific, Inc.) supplemented with 10\% fetal bovine serum (PAA Laboratories $\mathrm{GmbH}$; GE healthcare) at $37^{\circ} \mathrm{C}$ in a humidified atmosphere containing $5 \% \mathrm{CO}_{2}$. The pcDNA3.1(+)-Flag-ZNF545 plasmid was generated as previously described (6). To establish stably transfected cells with ectopic ZNF545 expression, the full-length expression plasmid pcDNA3.1(+)-Flag-ZNF545 was transfected into KYSE150 cells using Lipofectamine ${ }^{\circledR} 2000$ (Invitrogen; Thermo Fisher Scientific, Inc.) at a final concentration of $4 \mu \mathrm{g}$. The cells were then cultured and positively selected for resistance in $350 \mu \mathrm{g} / \mathrm{ml}$ G418 (Sigma-Aldrich; Merck KGaA) for 14 days and ZNF545 expression subsequently confirmed via semi-quantitative RT-PCR and western blotting.

DNA and RNA extraction. Total RNA was isolated from KYSE150 cells and esophageal tissues using TRIzol reagent (Invitrogen; Thermo Fisher Scientific, Inc.), whereas genomic DNA was extracted from KYSE150 cells and esophageal tissues using a QIAamp DNA Mini kit (Qiagen $\mathrm{GmbH}$ ) according to the manufacturer's protocol. DNA and RNA integrity were assessed using gel electrophoresis, and the nucleic acid samples were stored at $-80^{\circ} \mathrm{C}$ until required.

5-Aza-2'-deoxycytidine (Aza) and trichostatin A (TSA) treatment. KYSE150 cells were seeded into $100 \mathrm{~mm}$ dishes at a density of $1 \times 10^{5}$ cells $/ \mathrm{ml}$. Cells were first treated with $10 \mathrm{mmol} / \mathrm{l}$ of the demethylating reagent, Aza (Sigma-Aldrich; Merck $\mathrm{KGaA}$ ), for 3 days at $37^{\circ} \mathrm{C}$, before the addition of $100 \mathrm{nmol} / 1$ histone deacetylase inhibitor, TSA (Sigma-Aldrich; Merck KGaA), for a further $24 \mathrm{~h}$ at $37^{\circ} \mathrm{C}$ in a humidified atmosphere containing $5 \% \mathrm{CO}_{2}$, as described previously (5). RNA was subsequently collected from cells as aforementioned for the analysis of ZNF545 mRNA expression.

Semi-quantitative reverse transcription (RT)-PCR. RNA was quantified using the NanoDrop ND2000 spectrophotometer (NanoDrop Technologies; Thermo Fisher Scientific, Inc.). The A260/A280 nm ratio was calculated as between 1.6 and 1.9. Subsequently, cDNA was synthesized from $1 \mu \mathrm{g}$ total RNA using the reverse transcription (RT) system (Promega Corporation), and was stored at $-20^{\circ} \mathrm{C}$ until required. Semi-quantitative RT-PCR for ZNF545 was performed using treated/untreated KYSE150 cells as described previously (6). PCR products were amplified with 32 cycles for ZNF545 and 23 cycles for $\beta$-actin using the following temperature protocol: Initial denaturation at $95^{\circ} \mathrm{C}$ for $10 \mathrm{~min}$, denaturation at $95^{\circ} \mathrm{C}$ for $30 \mathrm{sec}$, annealing at $55^{\circ} \mathrm{C}$ for $30 \mathrm{sec}$, elongation at $72^{\circ} \mathrm{C}$ for $30 \mathrm{sec}$ and final extension at $25^{\circ} \mathrm{C}$ for $10 \mathrm{~min}$. Amplification was performed using Go-Taq ${ }^{\circledR}$ polymerase (Promega Corporation) and products were assayed on 2\% agarose gels. Samples were subsequently visualized and analyzed using the ChemiDoc MP system and Image Lab 3.0 software (each, Bio-Rad Laboratories, Inc.).

RT-quantitative PCR (RT-qPCR). GoTaq qPCR Master Mix (Promega Corporation) was used for RT-qPCR analysis, which was performed using a 7500 Real-Time PCR System (Applied Biosystems; Thermo Fisher Scientific, Inc.), following which melting-curve analysis was performed. Relative target gene expression was calculated using the $2^{-\Delta \Delta C a}$ method (7) with $\beta$-actin applied as the internal reference. The primer sequences are listed in Table I.

Bisulfite modification of DNA and methylation-specific PCR $(M S P)$. Bisulfite modification of DNA extracted from ESCC tissue and MSP were performed as described previously (8). DNA treated with bisulfite was amplified using MSP with methylation-specific (M1 and M2) or non-methylation-specific (U1 and U2) primer pairs. The primer sequences are listed in Table I. MSP was performed for 40 cycles with the following thermocycling conditions: Initial denaturation at $95^{\circ} \mathrm{C}$ for $10 \mathrm{~min}$, denaturation at $95^{\circ} \mathrm{C}$ for $30 \mathrm{sec}$, annealing at $58^{\circ} \mathrm{C}$ for $30 \mathrm{sec}$, elongation at $72^{\circ} \mathrm{C}$ for $30 \mathrm{sec}$ and final extension at $72^{\circ} \mathrm{C}$ for $10 \mathrm{~min}$. MSP was performed using AmpliTaq-Gold DNA Polymerase (Applied Biosystems; Thermo Fisher Scientific, Inc.) with annealing temperatures of $60^{\circ} \mathrm{C}$ for $\mathrm{M} 1 / \mathrm{M} 2$ and $58^{\circ} \mathrm{C}$ for $\mathrm{U} 1$ and $\mathrm{U} 2$. The MSP products were identified by performing gel electrophoresis with $2 \%$ agarose gels and a 100 bp DNA ladder as a marker (MBI Fermentas; Thermo Fisher Scientific, Inc.). The gels were visualized and analyzed as aforementioned.

Colony formation assay. KYSE150 cells stably transfected with pcDNA3.1(+)-Flag-ZNF545 or empty vector were seeded into a six-well plate at a density of 800 cells/well in culture media supplemented with G418. Following 14-21 days, cells were fixed using $4 \%$ paraformaldehyde for $30 \mathrm{~min}$ at room temperature and subsequently stained using $0.1 \%$ crystal violet solution for $15 \mathrm{~min}$ at room temperature. Colonies with $>50$ cells/colony were counted. The assay was performed three times for triplicate samples.

Flow cytometry analysis of apoptosis. To measure apoptotic cells, $2 \times 10^{5}$ cells were harvested with $0.1 \%$ trypsin from 6 -well plates at $48 \mathrm{~h}$ following transfection with the ZNF545 plasmid and empty controls, and centrifugation at $400 \mathrm{x}$ g for $5 \mathrm{~min}$ at room temperature. Apoptosis was measured by staining the cells with Annexin V-fluorescein isothiocyanate (FITC) and propidium iodide (PI; BD Biosciences) according to the manufacturer's protocol, and was analyzed using a Navios flow cytometer with Kaluza 2.0 software (Beckman Coulter, Inc.). All experiments were performed three times for triplicate samples.

Western blot analysis. At $48 \mathrm{~h}$ following transfection, cells were lysed using Mammalian Protein Extraction Reagent (Thermo Fisher Scientific, Inc.) supplemented with a phosphatase inhibitor cocktail (Sigma-Aldrich; Merck KGaA). 
Table I. List of primers used in the present study.

\begin{tabular}{llc}
\hline Primers & \multicolumn{1}{c}{ Sequences (5'-3') } & Product size (bp) \\
\hline ZNF545-F & GAGCCTTGGAAAGTTGTGAG & 245 \\
ZNF545-R & GGCATTTTCACACTACTGAAG \\
p53-F & TCAACAAGATGTTTTGCCAACTG \\
p53-R & ATGTGCTGTGACTGCTTGTAGATG & 118 \\
$\beta$-actin-F & CCTGTGGCATCCACGAAACT & 314 \\
$\beta$-actin-R & GAAGCATTTGCGGTGGACGAT \\
ZNF545-m1-F & TTTTTTTTAGGTTTTGTCGCGTC & 177 \\
ZNF545-m2-R & CTACTAAAAAAACCGAACGCG \\
ZNF545-u1-F & TTTTTTTTTAGGTTTTGTTGTGTT & 164 \\
ZNF545-u2-R & CCAAACACACTCACAAAATACA & \\
\hline
\end{tabular}

ZNF545, zinc finger protein 545; F, forward; R, reverse; m, methylation-specific; u, unmethylation specific.

Protein concentrations were determined using bicinchoninic acid Protein Assay Reagent (Thermo Fisher Scientific, Inc.). The protein samples were subsequently denatured and $40 \mu \mathrm{g}$ of each sample was separated by $10 \%$ SDS-PAGE before transferal onto PVDF membranes (Bio-Rad Laboratories, Inc.) (6). Following blocking with $5 \%$ non-fat milk at room temperature for $2 \mathrm{~h}$, and washing using TBS supplemented with $0.1 \%$ Tween-20, membranes were incubated overnight at $4^{\circ} \mathrm{C}$ with the following primary antibodies: ZNF545 (cat. no. sc-102235; 1:1,000; Santa Cruz Biotechnology, Inc.), Akt (cat. no. 4691; 1:1,000; Cell Signaling Technology, Inc.), phosphorylated (p)-Akt (cat. no. 4060; 1:2,000; Cell Signaling Technology, Inc.), p53 (cat. no. sc-126; 1:5,000; Santa Cruz Biotechnology, Inc.), BCL2 associated X, apoptosis regulator (Bax; cat. no. 5023; 1:1,000; Cell Signaling Technology, Inc.), p21 (cat. no. 2946; 1:2,000; Cell Signaling Technology, Inc.), cleaved-caspase 3 (cat. no. 9665; 1:1,000; Cell Signaling Technology, Inc.), cleaved-caspase 7 (cat. no. 9491; 1:1,000; Cell Signaling Technology, Inc.) and cleaved-caspase 9 (cat. no. 7237; 1:1,000; Cell Signaling Technology, Inc.), with $\beta$-actin (cat. no. LK-ab008-100; 1:200; Hangzhou Lianke Biology Technology Co., Ltd.) used as the internal loading control. Horseradish peroxidase (HRP)-conjugated anti-rabbit IgG (cat. no. 7074; 1:2,000; Cell Signaling Technology, Inc.) and HRP-conjugated anti-mouse IgG (cat. no. 7076; 1:2,000; Cell Signaling Technology, Inc.) secondary antibodies were added and incubated in a shaker at room temperature for $60 \mathrm{~min}$. Protein blots were visualized using an enhanced chemiluminescence kit (Amersham Pharmacia Biotech Ltd.; GE Healthcare) and imaged and analyzed using the LAS-4000 Imaging System (Medical Systems; Fujifilm).

Statistical analysis. Statistical analysis was performed using SPSS software (version 13.0; SPSS, Inc.). All data were presented as the mean \pm standard deviation and analyzed using Student's t-test, Wilcoxon signed-rank tests and Pearson's chi-squared tests as appropriate. All experiments were repeated in triplicate and $\mathrm{P}<0.05$ was considered to indicate a statistically significant difference.

\section{Results}

Promoter methylation downregulates ZNF545 expression in ESCC tissues. The expression of ZNF545 mRNA in tumor and paired adjacent normal tissues from 15 patients diagnosed with ESCC was examined using RT-qPCR. ZNF545 mRNA was significantly downregulated in ESCC tumors when compared with adjacent normal tissues $(\mathrm{P}=0.022$; Fig. 1A). ZNF545 expression was also restored following the pharmacologic treatment of Aza and TSA in KYSE150 (Fig. 1B). The promoter methylation status of ZNF545 in tumor tissues $(n=64)$ and adjacent normal tissues $(n=64)$ was also evaluated using an MSP assay. ZNF545 promoter methylation was observed in $76.6 \%(49 / 64)$ of primary tumors, compared with only $28.1 \%(28 / 64)$ of adjacent normal tissues $(\mathrm{P}<0.001$; Fig. $1 \mathrm{C}$ and Table II). Lastly, the association between ZNF545 methylation and clinicopathological features in ESCC patients was investigated, but no significant association was observed for age, sex, tumor location, staging, pathologic grade or lymph node metastasis $(\mathrm{P}>0.05$ for all features; Table III).

ZNF545 transfection inhibits cell clonogenicity and induces apoptosis in ESCC cells. ZNF545 function in ESCC was evaluated by ectopically overexpressing ZNF545 in the ESCC cell line, KYSE150 (Fig. 2A). The effect of ZNF545 expression on KYSE150 cell proliferation was subsequently determined using a colony formation assay. The total number of colonies was significantly decreased in cells stably transfected with the ZNF545 vector $(\mathrm{P}<0.001)$ compared with those transfected with the control vector (Fig. 2B). The effects of ectopic ZNF545 expression on apoptosis was then examined in KYSE150 cells using annexin V-FITC/PI double-staining. A significant increase in the number of apoptotic cells was observed in the ZNF545 overexpression group when compared with controls $(\mathrm{P}<0.001$; Fig. $2 \mathrm{C})$. In support of this, a marked increase in cleaved caspases 3, 7 and 9 protein expression levels were also observed in KYSE150 cells ectopically expressing ZNF545 when compared with those transfected with the control vector (Fig. 2D). 
Table II. Promoter methylation status of ZNF545 in esophageal cancer.

\begin{tabular}{lcccr}
\hline & & \multicolumn{2}{c}{ ZNF545 promoter } & \\
\cline { 3 - 4 } Tissues & Samples (n) & Methylated & Unmethylated & Frequency of methylation (\%) \\
\hline Esophageal tumors & 64 & 49 & 15 & $76.6^{\mathrm{a}}$ \\
Adjacent esophageal tissues & 64 & 18 & 46 & 28.1 \\
\hline
\end{tabular}

${ }^{a} \mathrm{P}<0.001$ vs. adjacent esophageal tissues. $\mathrm{P}<0.001$ (Pearson's chi-squared test). ZNF, zinc finger protein 545 .

Table III. Association between ZNF545 methylation and the clinicopathological features of patients with esophageal cancer.

\begin{tabular}{|c|c|c|c|c|}
\hline \multirow[b]{2}{*}{ Clinicopathologic feature } & \multirow[b]{2}{*}{ Number $(n=64)$} & \multicolumn{2}{|c|}{ ZNF545 methylation status } & \multirow[b]{2}{*}{ P-value } \\
\hline & & Methylated & Unmethylated & \\
\hline Age & & & & 0.447 \\
\hline$\leq 60$ & 23 & 19 & 4 & \\
\hline$>60$ & 38 & 27 & 11 & \\
\hline Unknown & 3 & 3 & 0 & \\
\hline Sex & & & & 0.731 \\
\hline Male & 51 & 39 & 12 & \\
\hline Female & 10 & 7 & 3 & \\
\hline Unknown & 3 & 3 & 0 & \\
\hline Tumor location & & & & 0.596 \\
\hline Upper & 13 & 9 & 4 & \\
\hline Median & 35 & 26 & 9 & \\
\hline Lower & 24 & 20 & 4 & \\
\hline Unknown & 4 & 4 & 0 & \\
\hline Tumor stage & & & & 0.613 \\
\hline I-II & 27 & 22 & 5 & \\
\hline III-IV & 19 & 13 & 6 & \\
\hline Unknown & 18 & 14 & 4 & \\
\hline Pathologic grade & & & & 0.158 \\
\hline G1 & 25 & 22 & 3 & \\
\hline G2 & 37 & 24 & 13 & \\
\hline G3 & 11 & 7 & 4 & \\
\hline Unknown & 6 & 4 & 2 & \\
\hline Lymph node metastasis & & & & 0.368 \\
\hline Positive & 13 & 8 & 5 & \\
\hline Negative & 48 & 38 & 10 & \\
\hline Unknown & 3 & 3 & 0 & \\
\hline
\end{tabular}

ZNF545, zinc finger protein 545. Certain data could not be provided due to a loss of follow-up. There are various overlaps for tumor location and pathologic grade between patients.

ZNF545 suppresses tumors via the transcriptional activation of p53 or a p53-independent signaling pathway in p53-mutant KYSE150 cells. In a previous study, ZNF545 was identified as a novel tumor suppressor that inhibited cell proliferation and colony formation, and induced apoptosis via TP53 reactivation in multiple myeloma (6). The association between ZNF545 and the p53 signaling pathway was examined as a consequence of the potential tumor suppressive function of ZNF545 revealed in ESCC. RT-qPCR analysis revealed that ectopic ZNF545 expression in KYSE150 cells upregulated p53 mRNA expression when compared with those transfected with the control vector $(\mathrm{P}<0.05$; Fig. $3 \mathrm{~A})$, suggesting that ZNF545 may activate p53 transcription. The protein expression levels of two downstream effectors of the p53 pathway, 
A

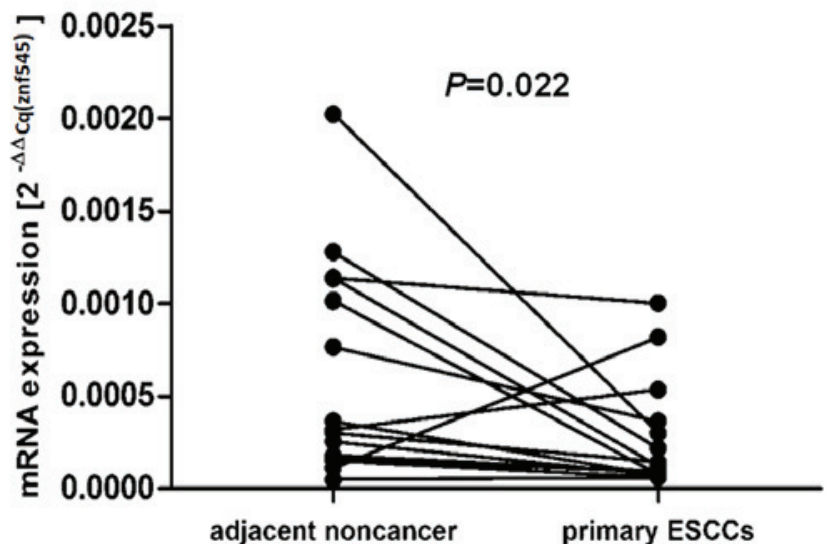

B

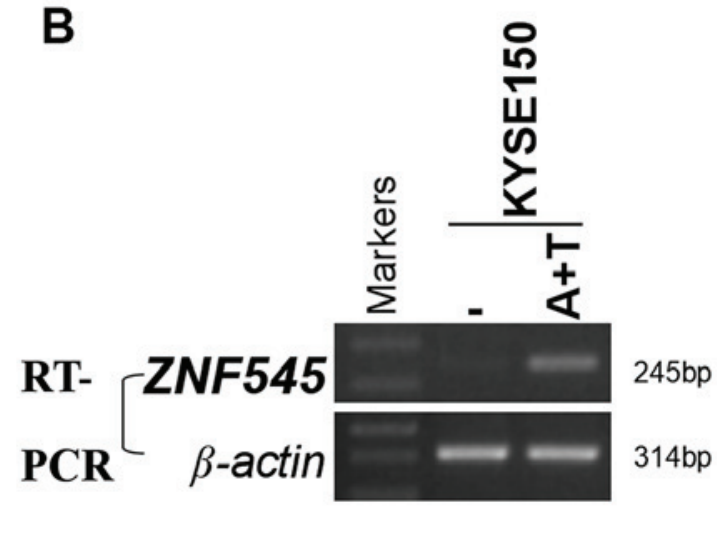

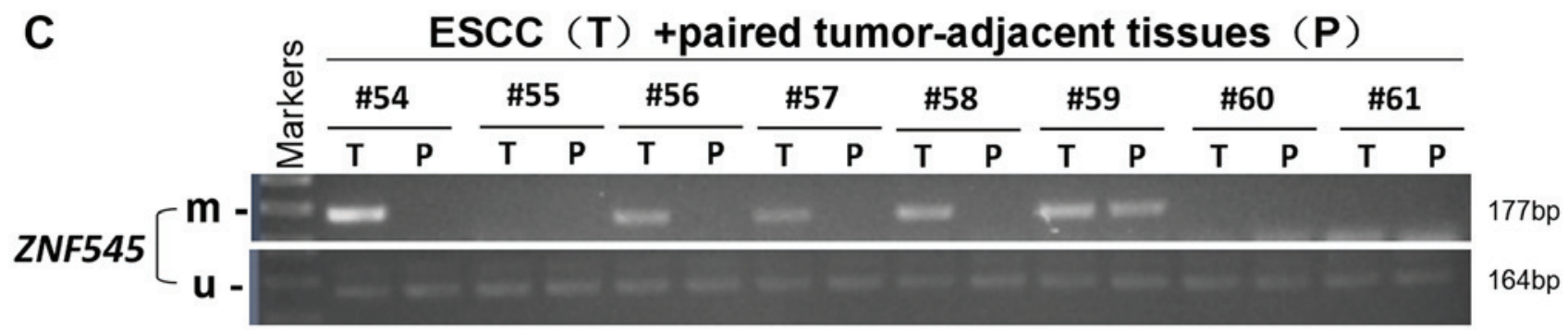

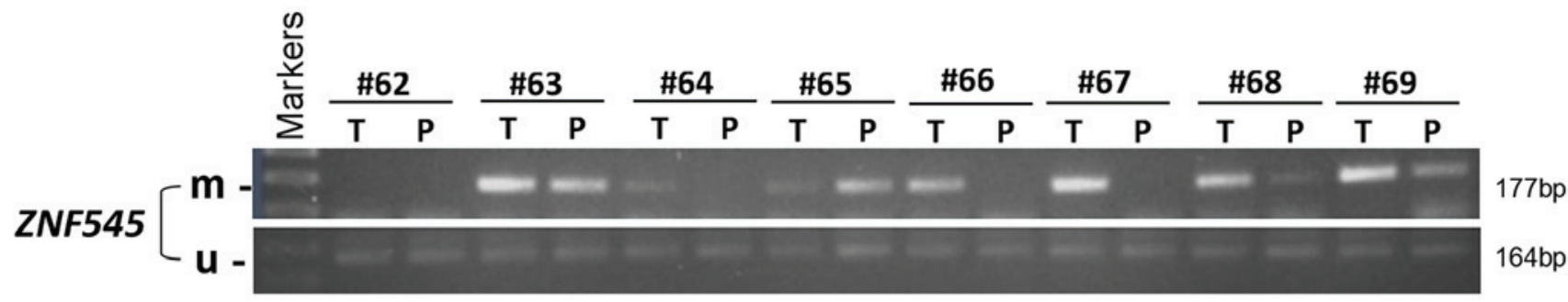

Figure 1. ZNF545 expression is downregulated by methylation in ESCC tissues. (A) ZNF545 mRNA expression was significantly reduced in primary ESCC tissues when compared with adjacent noncancer samples $(n=15)$, according to reverse transcription-quantitative PCR analysis. (B) ZNF545 mRNA expression was restored in KYSE150 cells following treatment with the demethylating reagent, 5-Aza-2'-deoxycytidine, and the histone deacetylase inhibitor, trichostatin A. (C) Representative images showing promoter methylation of ZNF545 in ESCC tissues compared with paired tumor-adjacent tissues using methylation-specific PCR. $\beta$-actin was used as an internal control. ZNF545, zinc finger protein 545; ESCC, esophageal squamous cell carcinoma; m, methylated; u, unmethylated; A + T, 5-Aza-2'-deoxycytidine and trichostatin A.

Bax and p21, were increased in KYSE150 p53-mutant cells transfected with ZNF545; whereas the expression of Akt was not affected by ZNF545 transfection (Fig. 3C). Although ZNF545 may exert its tumor suppressive effects in ESCC by activating p53 transcription through a p53-dependent signaling pathway, intracellular signaling remains unclear. Furthermore, upregulating p21 and Bax expression via p53-independent signaling pathways in tumor cells with mutant p53 is another possibility (Fig. 3B).

\section{Discussion}

Previous studies have identified ZNF545 as a functional tumor suppressor gene in a number of solid tumors, including ESCC, and its expression is frequently silenced by promoter methylation $(4,5,9,10)$. However, the association between p53 and ZNF545, and its downstream functions in p53-mutant ESCC remain unclear. The present study provides novel evidence for an association between ZNF545 and mutant p53 in ESCC. To assess ZNF545 methylation as a potential epigenetic biomarker, its relevance in relation to the clinicopathological features of ESCC was also evaluated, as aberrant DNA methylation has been reported to mediate tumor suppressor gene silencing and consequent tumorigenesis (11). As demonstrated by MSP analysis in the present study, ZNF545 methylation was detected in $76.6 \%$ of ESCC tissues when compared with $28.1 \%$ of adjacent normal tissues. However, no significant association between the clinicopathological features of patients with ESCC and the methylation status of ZNF545 was observed. In general, bisulfite sequencing PCR (BSP) is applied to discover methylation sites on genes, and MSP primers are subsequently designed according to the methylation sites to determine the optimal conditions and methods to detect methylation. BSP can yield accurate results due to subsequent sequencing analysis; however, a high number of clones are required for this, making the procedure more difficult to operate in large quantities. MSP can be used to screen a large number of specimens for the detection of methylation status. Therefore, the MSP was selected to evaluate ZNF545 methylation in clinical samples in the present study. A previous study demonstrated that ZNF545 methylation was associated with survival in patients with ESCC (5), and poor survival in gastric and hepatic cancer $(12,13)$. The present 
A

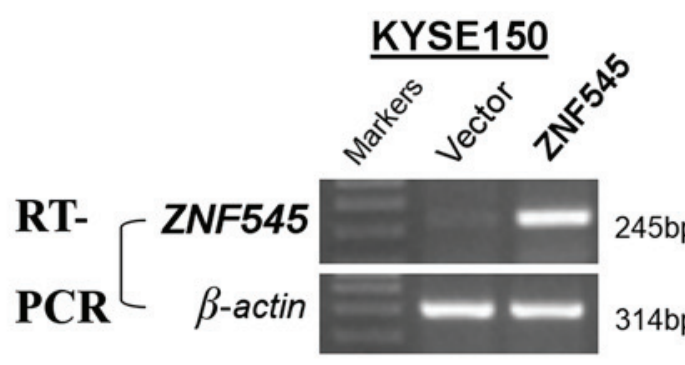

C

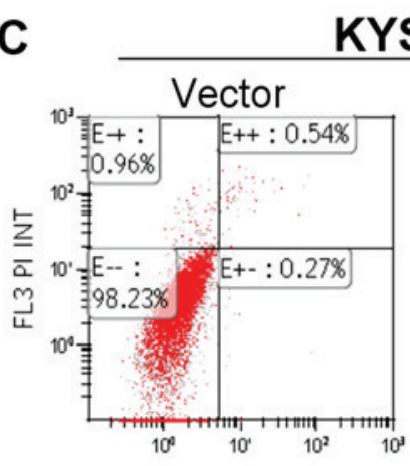

FL1 Annexin V-FITCINT
KYSE150

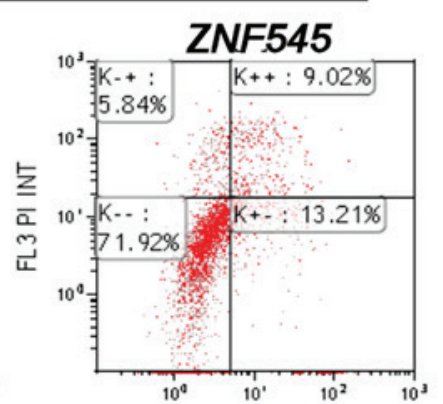

FL1 Annexin V-FITC INT
B
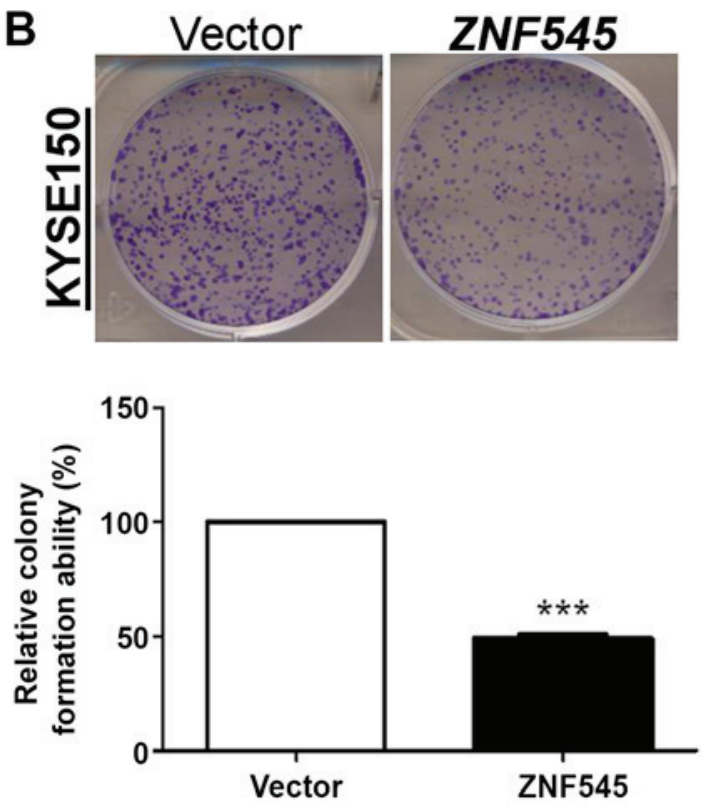

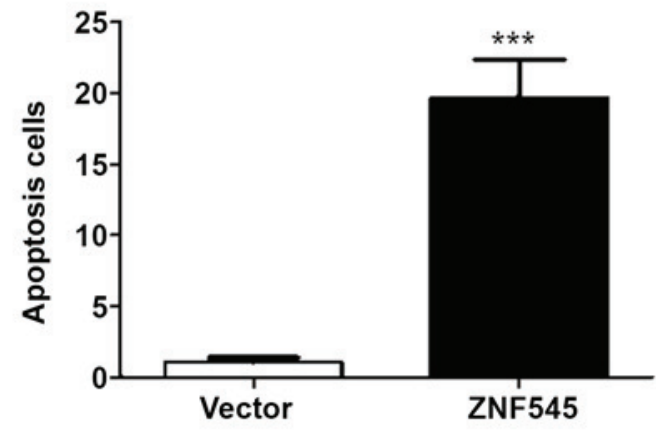

D

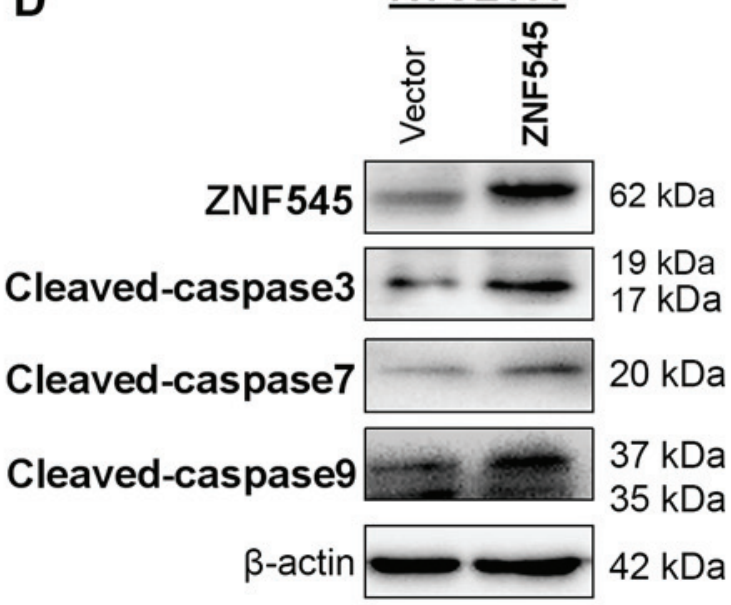

Figure 2. ZNF545 inhibits cell growth by suppressing colony formation and inducing apoptosis in KYSE150 cells. (A) Expression of ZNF545 mRNA in KYSE150 cells transfected with empty vector or ZNF545 overexpression plasmids by semi-quantitative-reverse transcription PCR. (B) Representative images of colony formation and quantitative analysis of colonies. The histogram shows the number of colonies in the ZNF545 group relative to the control group (100\%). (C) Apoptosis of KYSE150 cells was examined by staining cells with Annexin V-FITC and PI followed by flow cytometry analysis. (D) Cleaved-caspase 3 , cleaved-caspase 7 and cleaved-caspase 9 levels were evaluated in vector- and ZNF545-transfected KYSE150 cells by western blotting analysis. $\beta$-actin was used as an internal control. ${ }^{* * *} \mathrm{P}<0.001$ vs. Vector. ZNF545, zinc finger protein 545; FITC, fluorescein isothiocyanate; PI, propidium iodide; bp, base pairs; ESCC, esophageal squamous cell carcinoma; FL1 and FL3, the channels for PI and FITC respectively; INT, the moderate expression of fluorescence.

study also revealed that ectopic ZNF545 expression inhibited cell growth by significantly suppressing colony formation and inducing apoptosis in ESCC cells.

The biological function of p53 in tumor suppression is well established (14). p53 activates downstream mediators of cell proliferation inhibition (p21 and growth arrest and DNA damage inducible- $\alpha$ ) and pro-apoptotic proteins (Bax and PUMA) resulting in cell cycle arrest and apoptosis $(15,16)$. In addition, the zinc finger protein, JAZ, has been reported to serve a role in mediating tumor growth by regulating $\mathrm{p} 53$ activity (17). Transcriptional activation of TP53 by ZNF545 has also been reported in breast cancer (10). Although activation of the MDM2 ubiquitin-ligase by Akt and subsequent p53 degradation have been implicated in tumorigenesis $(18,19)$, no changes in Akt expression following ectopic ZNF545 expression was observed in ESCC cells in the present study, which is consistent with previous observations in multiple myeloma (6). However, p53 mutations are more commonly observed in malignancies involving organs compared with their hematological counterparts (20). Strategies targeting mutant p53, and altered signaling pathways exhibited in p53-mutant cells have been evaluated with the aim of uncovering novel treatments for malignancies associated with mutant p53 (20).

The results of the present study suggest that ZNF545 may transcriptionally activate p53 and upregulate the protein expression of its potential downstream effectors, p21 and Bax, in the p53-mutant KYSE150 ESCC cell line. As the functional status of p53 in KYSE150 cells is unknown, these observations 


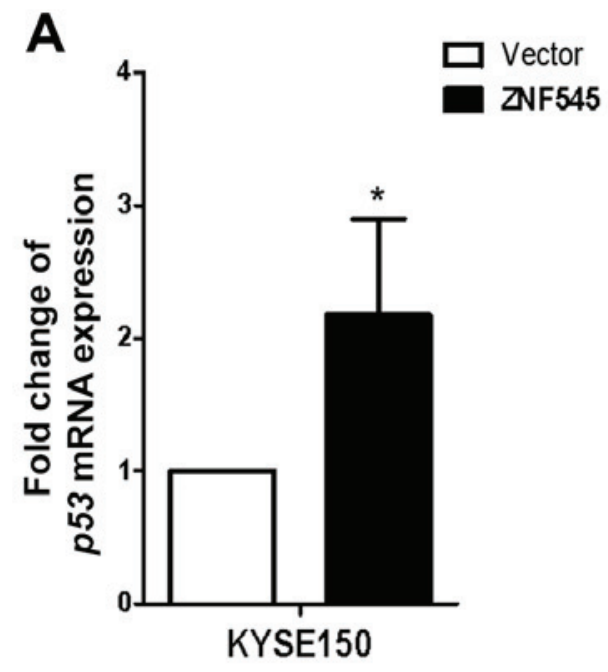

B

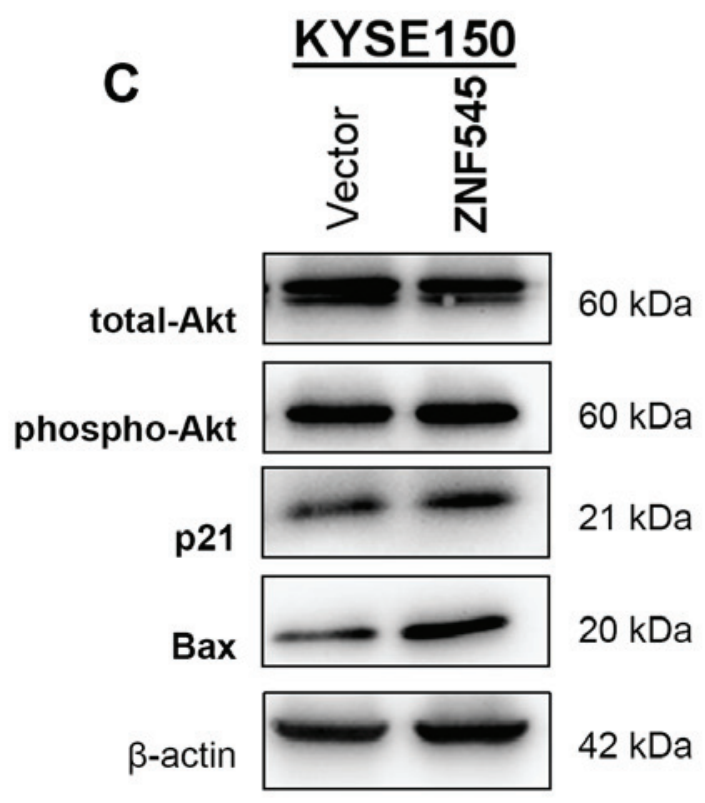

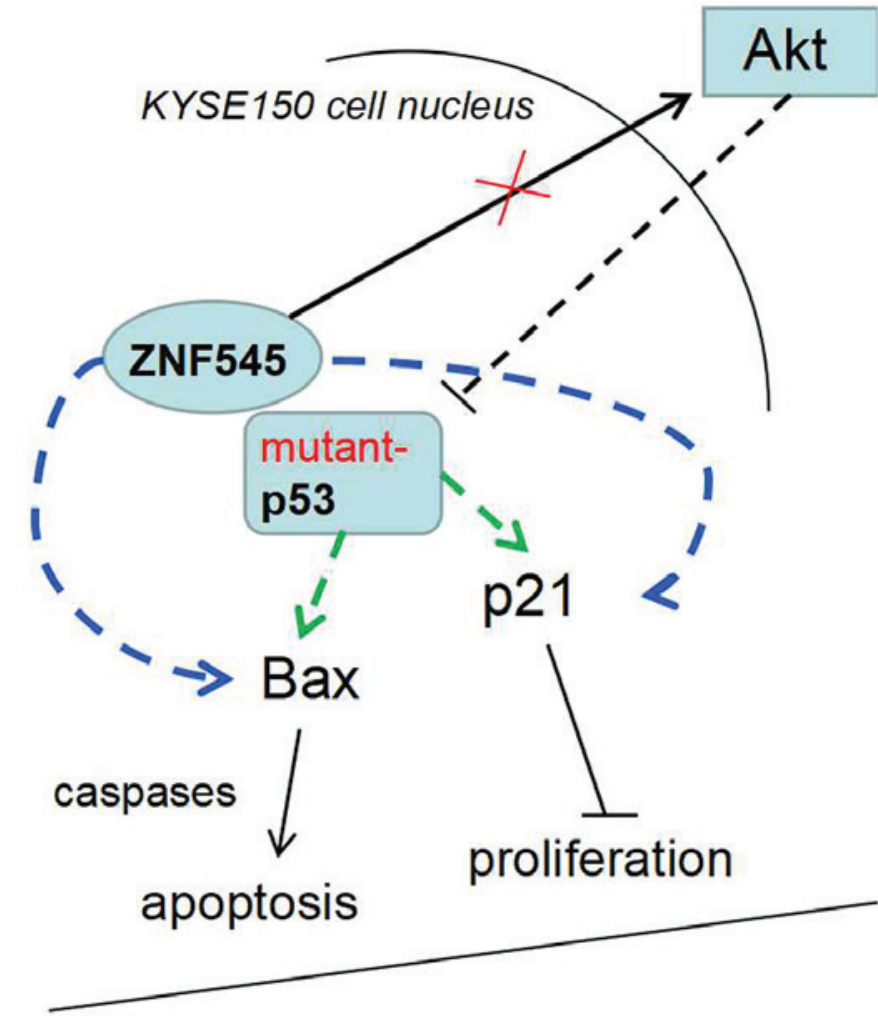

Inhibiting Tumor Growth

\author{
Note \\ The green dotted line: p53-dependent \\ The blue dotted line: p53-independent
}

Figure 3. ZNF545 transcriptionally activates p53 or induces p21 and Bax through p53-independent signaling pathways in the p53-mutant KYSE150 cell line. (A) Transcriptional activation of p53 following ectopic expression of ZNF545 in KYSE150 cells, as determined using reverse transcription-quantitative PCR. (B) Proposed mechanism of the tumor suppressive function of ZNF545 in KYSE150 cells through p53 transcriptional activation via a p53-dependent or independent signaling pathway to induce p21 and Bax expression. (C) Western blotting analysis was performed in KYSE150 cells transfected with empty vector or ZNF545 overexpression plasmids, using antibodies against total-Akt, phospho-Akt, p53, p21 and Bax. $\beta$-actin was used as an internal control. ${ }^{*} \mathrm{P}<0.05$ vs. Vector. ZNF545, zinc finger protein 545; Bax, BCL2 associated X, apoptosis regulator; phospho-Akt, phosphorylated Akt.

indicate that mutant $\mathrm{p} 53$ proteins in ESCC may retain the ability to activate p21, but also suggests that ZNF545-mediated tumor suppression may be independent of p53 (21), thus providing insights into a potentially novel tumorigenic mechanism in ESCC. Indeed, p21 can be regulated by p53-dependent and p53-independent pathways (22). However, the mechanism by which ZNF545 may upregulate p21 and other effectors in the absence of p53 warrants further evaluation. One hypothesis is that the function of mutant p53 could be restored by specific DNA binding or transcriptional transactivation via a p53-dependent pathway (23). Alternatively, it is possible that p21 and Bax may be upregulated by ZNF545 through an as yet undiscovered p53-independent signaling pathway, such as the nuclear factor- $\kappa \mathrm{B}$ signaling pathway (24). A further in-depth investigation of the role of ZNF545 in mutant-p53 tumors, and the potential crosstalk with other signaling pathways, will need to be performed to support the results of the present study.

In conclusion, the anti-tumor effects of ZNF545 in a p53-mutant ESCC cell line may be mediated by the transcriptional activation of $\mathrm{p} 53$ or by the induction of $\mathrm{p} 21$ and Bax by an as yet unknown p53-independent signaling pathway. In addition, activation of these potential signaling pathways during tumorigenesis may be inhibited by ZNF545 methylation. Tumor-specific methylation of ZNF545 may be a potential epigenetic biomarker for the early diagnosis of ESCC. However, data in the present study is limited by the lack of additional ESCC cell lines harboring other mutant-p53 genotypes to confirm these findings. In addition, no other ESCC cell line with upregulated expression was available to perform a ZNF545 knockdown assay. 


\section{Acknowledgements}

Not applicable.

\section{Funding}

The present study was supported by the Research Foundation of Southwest Medical University for Youth (grant no. 090300030685), the Funded Project of Affiliated Hospital of Southwest Medical University for Doctors (grant no. 17135), the Health and Family Planning Commission of Sichuan Province (grant no. 17PJ557) and the National Natural Science Foundation of China (grant no. 81201682).

\section{Availability of data and materials}

The datasets used and/or analyzed during the present study are available from the corresponding author on reasonable request.

\section{Authors' contributions}

YF designed this study, contributed to the experiments, and drafted the manuscript. YW contributed to the experiments and interpreted the results. SF performed the data analysis. DL and SL contributed to the acquisition of clinical samples. All authors contributed to the revisions of this manuscript and approved the final draft.

\section{Ethics approval and consent to participate}

Ethics approval was received from the Affiliated Hospital of Southwest Medical University. All participants provided written consent during their enrollment.

\section{Patient consent for publication}

All patients provided written consent during enrollment for publication.

\section{Competing interests}

The authors declare that they have no competing interests.

\section{References}

1. Murphy G, McCormack V, Abedi-Ardekani B, Arnold M, Camargo MC, Dar NA, Dawsey SM, Etemadi A, Fitzgerald RC, Fleischer DE, et al: International cancer seminars: A focus on esophageal squamous cell carcinoma. Ann Oncol 28: 2086-2093, 2017.

2. Abnet CC, Arnold M and Wei WQ: Epidemiology of esophageal squamous cell carcinoma. Gastroenterology 154: 360-373, 2018.

3. Holubekova V, Mendelova A, Jasek K, Mersakova S, Zubor P and Lasabova Z: Epigenetic regulation by DNA methylation and miRNA molecules in cancer. Future Oncol 13: 2217-2222, 2017.

4. Cheng Y, Liang P, Geng H, Wang Z, Li L, Cheng SH, Ying J, Su X, Ng KM, Ng MH, et al: A novel 19q13 nucleolar zinc finger protein suppresses tumor cell growth through inhibiting ribosome biogenesis and inducing apoptosis but is frequently silenced in multiple carcinomas. Mol Cancer Res 10: 925-936, 2012.

5. Ye L, Xiang T, Fan Y, Zhang D, Li L, Zhang C, He X, Xiang Q, Tao Q and Ren G: The 19q13 KRAB Zinc-finger protein ZFP82 suppresses the growth and invasion of esophageal carcinoma cells through inhibiting NF- $\kappa \mathrm{B}$ transcription and inducing apoptosis. Epigenomics 11: 65-80, 2019.
6. Fan Y, Zhan Q, Xu H, Li L, Li C, Xiao Q, Xiang S, Hui T, Xiang T and Ren G: Epigenetic identification of ZNF545 as a functional tumor suppressor in multiple myeloma via activation of p53 signaling pathway. Biochem Biophys Res Commun 474: 660-666, 2016 .

7. Livak KJ and Schmittgen TD: Analysis of relative gene expression data using real-time quantitative PCR and the 2(-Delta Delta C(T)) method. Methods 25: 402-408, 2001.

8. Tao Q, Huang H, Geiman TM, Lim CY, Fu L, Qiu GH and Robertson KD: Defective de novo methylation of viral and cellular DNA sequences in ICF syndrome cells. Hum Mol Genet 11: 2091-2102, 2002.

9. Wang S, Cheng Y, Du W, Lu L, Zhou L, Wang H, Kang W, Li X, Tao Q, Sung JJ and Yu J: Zinc-finger protein 545 is a novel tumour suppressor that acts by inhibiting ribosomal RNA transcription in gastric cancer. Gut 62: 833-841, 2013.

10. Xiao Y, Xiang T, Luo X, Li C, Li Q, Peng W, Li L, Li S, Wang Z, Tang L, et al: Zinc-finger protein 545 inhibits cell proliferation as a tumor suppressor through inducing apoptosis and is disrupted by promoter methylation in breast cancer. PLoS One 9: e110990, 2014.

11. Herman JG and Baylin SB: Gene silencing in cancer in association with promoter hypermethylation. N Engl J Med 349: 2042-2054, 2003.

12. Deng J, Liang H, Ying G, Dong Q, Zhang R, Yu J, Fan D and Hao X: Poor survival is associated with the methylated degree of zinc-finger protein 545 (ZNF545) DNA promoter in gastric cancer. Oncotarget 6: 4482-4495, 2015.

13. Yu J, Li X, Tao Q, Yu XL, Cheng ZG, Han ZY, Guo M and Liang P: Hypermethylation of ZNF545 is associated with poor prognosis in patients with early-stage hepatocellular carcinoma after thermal ablation. Gut 64: 1836-1837, 2015.

14. Kaiser AM and Attardi LD: Deconstructing networks of p53-mediated tumor suppression in vivo. Cell Death Differ 25: 93-103, 2018

15. Shu KX, Li B and Wu LX: The p53 network: P53 and its downstream genes. Colloids Surf B Biointerfaces 55: 10-18, 2007.

16. Ozaki T, Nakagawara A and Nagase H: RUNX Family participates in the regulation of p53-dependent DNA damage response. Int J Genomics 2013: 271347, 2013.

17. Yang M, Wu S, Su X and May WS: JAZ mediates G1 cell-cycle arrest and apoptosis by positively regulating p 53 transcriptional activity. Blood 108: 4136-4145, 2006.

18. Gottlieb TM, Leal JF, Seger R, Taya Y and Oren M: Cross-talk between Akt, p53 and Mdm2: Possible implications for the regulation of apoptosis. Oncogene 21: 1299-1303, 2002.

19. Janicke RU, Sohn D and Schulze-Osthoff K: The dark side of a tumor suppressor: Anti-apoptotic p53. Cell Death Differ 15: 959-976, 2008

20. Zhao D, Tahaney WM, Mazumdar A, Savage MI and Brown PH: Molecularly targeted therapies for p53-mutant cancers. Cell Mol Life Sci 74: 4171-4187, 2017.

21. Andries V, Vandepoele K, Staes K, Berx G, Bogaert P, Van Isterdael G, Ginneberge D, Parthoens E, Vandenbussche J, Gevaert K and van Roy F: NBPF1, a tumor suppressor candidate in neuroblastoma, exerts growth inhibitory effects by inducing a G1 cell cycle arrest. BMC Cancer 15: 391, 2015.

22. Mirzayans R, Andrais B, Kumar P and Murray D: Significance of Wild-type p53 signaling in suppressing apoptosis in response to chemical genotoxic agents: Impact on chemotherapy outcome. Int J Mol Sci 18: pii: E928, 2017.

23. Selivanova $\mathrm{G}$ and Wiman KG: Reactivation of mutant $\mathrm{p} 53$ : Molecular mechanisms and therapeutic potential. Oncogene 26: 2243-2254, 2007.

24. Basile JR,Eichten A,Zacny V andMüngerK:NF-kappaB-mediated induction of $\mathrm{p} 21$ (Cip1/Waf1) by tumor necrosis factor alpha induces growth arrest and cytoprotection in normal human keratinocytes. Mol Cancer Res 1: 262-270, 2003.

This work is licensed under a Creative Commons Attribution-NonCommercial-NoDerivatives 4.0 International (CC BY-NC-ND 4.0) License. 